\title{
ANEM: A rotating composite target to produce an atmospheric-like neutron beam at the LNL SPES facility
}

\author{
Gabriela Carolina Acosta Urdaneta \\ Dept. of Physics and Astronomy, University of Padova, via Marzolo 8, I-35131 Padova, Italy \\ and INFN Padova, via Marzolo 8, I-35131 Padova, Italy \\ gabriela.acosta@lnl.infn.it \\ Dario Bisello \\ Dept. of Physics and Astronomy, University of Padova, via Marzolo 8, I-35131 Padova, Italy \\ and INFN Padova, via Marzolo 8, I-35131 Padova, Italy \\ dario.bisello@pd.infn.it \\ Juan Esposito \\ INFN Legnaro, 35020, Legnaro (Pd), Italy \\ juan.esposito@lnl.infn.it \\ Pierfrancesco Mastinu \\ INFN Legnaro, 35020, Legnaro (Pd), Italy \\ pierfrancesco.mastinu@lnl.infn.it \\ Gianfranco Prete \\ INFN Legnaro, 35020, Legnaro (Pd), Italy \\ gianfranco.prete@lnl.infn.it \\ Luca Silvestrin \\ Dept. of Physics and Astronomy, University of Padova, via Marzolo 8, I-35131 Padova, Italy \\ and INFN Padova, via Marzolo 8, I-35131 Padova, Italy \\ luca.silvestrin@pd.infn.it \\ Jeffery Wyss \\ DICeM, University of Cassino, via Di Biasio 43, I-03043 Cassino (Fr), Italy \\ and INFN Padova, via Marzolo 8, I-35131 Padova, Italy \\ wyss@unicas.it
}

Published 1 September 2016

A fast neutron $(\mathrm{E}>1 \mathrm{MeV})$ irradiation facility is under development at the $70 \mathrm{MeV}$ SPES proton cyclotron at LNL (Legnaro, Italy) to investigate neutron-induced Single Event Effects (SEE) in microelectronic devices and systems. After an overview on neutron-induced SEE in electronics,

This is an Open Access article published by World Scientific Publishing Company. It is distributed under the terms of the Creative Commons Attribution 3.0 (CC-BY) License. Further distribution of this work is permitted, provided the original work is properly cited. 
we report on the progress in the design of ANEM (Atmospheric Neutron EMulator), a water-cooled rotating target made of $\mathrm{Be}$ and $\mathrm{W}$ to produce neutrons with an energy spectrum similar to that of neutrons produced by cosmic rays at sea-level. In ANEM, the protons from the cyclotron alternatively impinge on two circular sectors of $\mathrm{Be}$ and $\mathrm{W}$ of different areas; the effective neutron spectrum is a weighted combination of the spectra from the two sectors. In this contribution, we present the results of thermal-mechanical Finite Element Analysis (ANSYS) calculations of the performance of the ANEM prototype. The calculations at this stage indicate that ANEM can deliver fast neutrons with an atmospheric-like energy spectrum and with an integral flux $\Phi_{\mathrm{n}}(1-70 \mathrm{MeV}) \sim 10^{7} \mathrm{n} \mathrm{cm}^{-2} \mathrm{~s}^{-1}$ that is $3 \times 10^{9}$ more intense than the natural one at sealevel: a very competitive flux for SEE testing.

Keywords: Radiation damage; neutron facility; neutron source.

PACS numbers: $61.80 . \mathrm{Hg}, 61.82 . \mathrm{Fk}, 29.25 . \mathrm{Dz}$.

\section{Introduction: Neutron-induced Effects in Microelectronics at LNL}

The study of radiation damage in electronics is an important field of scientific and technological research: radiation tolerance is essential for many scientific and technological applications of electronics in hostile radiation environments such as High Energy Physics (HEP), Nuclear Reactors, Nuclear Medicine and Space Applications. However, certain types of damage caused by neutrons produced by cosmic rays in the atmosphere are of growing concern regarding the use of state-of-the-art electronics highreliability applications. ${ }^{1}$

A Single Event Effect (SEE) in electronics occurs when a single particle generates enough unwanted charge to cause an instantaneous anomalous device or system behavior. Neutrons induce SEE indirectly when they interact with the nuclei of the semiconductor materials and surroundings to produce highly ionizing particles. Neutron-induced SEE types range from Soft Errors such as Single Event Upsets (SEU) that consist in memory data corruption or changes in the state of logic circuits to catastrophic Hard Errors that may permanently damage a device such as Single Event Latch-up in CMOS technologies and Single Event Burn-out in power MOSFETs.

The neutron SEE sensitivity of a device, for instance a memory circuit, is determined experimentally by detecting and counting anomalous events in a known neutron field. The sensitivity of a device is expressed in $\mathrm{cm}^{2}$ as an effective cross section and is a function of the energy of the impinging neutrons: $\sigma(E)=N_{S E E} /\left(\phi(E) \cdot N_{b i t s}\right)$, where $N_{S E E}$ is the number of SEE observed in the device, $\phi(E)$ is the fluence of neutrons with energy $E$ and $N_{\text {bits }}$ is the number of memory bits. Typically, for a given device, an SEE may occur only if the energy of the impinging neutron is above some minimum threshold value $\mathrm{E}_{0}$. The energy dependent cross-section $\sigma(\mathrm{E})$ is a Weibull-like function that increases from $\mathrm{E}_{0}$ with neutron energy until a plateau value $\sigma_{\mathrm{P}}$ is reached. A typical reference value of the plateau cross-section per bit for SEU is $\sigma_{\mathrm{P}}=10^{-14} \mathrm{~cm}^{2} / \mathrm{bit}$, the minimum reference value for the number of bits is $\mathrm{N}_{\text {bits }}=1 \mathrm{~GB}$ hence the corresponding SEU plateau cross-section per device is $\sigma_{\mathrm{P}}=8 \times 10^{-5} \mathrm{~cm}^{2} /$ device. 


\section{Atmospheric Neutrons and SEE Testing}

The issues and concerns of SEE induced by fast neutrons $(\mathrm{E}>1 \mathrm{MeV})$ that have always been crucial in avionics are expanding quickly to include ground-based electronics. Vital instrumentation, both onboard high altitude flights and on the ground, from mountains to sea-level, are constantly exposed to so-called atmospheric neutron produced by high energy cosmic rays in the upper atmosphere. Compared to high-altitude flights, the probability at sea-level of SEE induced by atmospheric neutrons is much lower, but the fast growing number of electronic devices and systems used in ever more diverse groundbased applications is much larger. Atmospheric neutrons have a continuous energy spectrum with maximum energy exceeding $1 \mathrm{GeV}$, but the flux at sea level is low. The integral flux of atmospheric neutrons with $\mathrm{E}>1 \mathrm{MeV}$ is $21 \mathrm{n} \mathrm{cm}^{-2} \mathrm{hr}^{-1}$, with two-thirds of them in the 1-70 MeV energy range (at flight altitudes the integral flux is about 300 times more intense). ${ }^{2}$ The reference device mentioned above would show a rate of neutron-induced SEU equal to $\mathrm{R}_{\mathrm{SEU}} \sim 15$ events/device per year.

Research groups and industries that study neutron-induced SEE and validate electronics use large, high-energy spallation facilities like LANSCE and ISIS, where 800 $\mathrm{MeV}$ protons on thick (proton stopping) heavy targets produce high-flux atmospheric-like neutron beams in the $1-800 \mathrm{MeV}$ neutron energy range. The neutron fluxes at the SEE test stations at LANSCE (Ice House) and ISIS (Chip-IR, completed recently) are, respectively, $\mathrm{F}=10^{8}$ and $\mathrm{F}=10^{9}$ times higher than the natural one at sea-level (Fig. 1). ${ }^{3,4}$

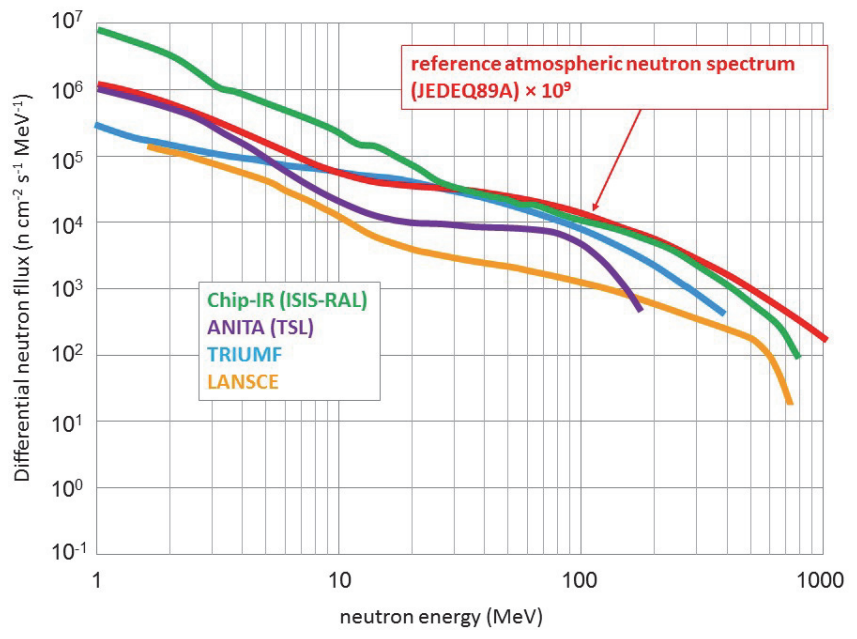

Fig. 1. The differential energy spectra of neutrons at irradiation facilities. The indicated curve is the JEDEC reference sea-level atmospheric neutron spectrum multiplied by $\mathrm{F}=10^{9}$.

An acceleration factor of $\mathrm{F}=1 \times 10^{8}$ (corresponding to an integral neutron flux of $\left.\Phi_{\mathrm{n}}(\mathrm{E}>1 \mathrm{MeV}) \sim 6 \times 10^{5} \mathrm{n} \mathrm{cm}^{-2} \mathrm{~s}^{-1}\right)$ implies that one hour of irradiation is equivalent to $\sim 10^{4}$ years of exposure at sea level. The push from the electronics industry to test at higher 
acceleration factors is due to the expected increase in numbers of devices in a fast growing electronics market. The increased interest in neutron effects in ground-based electronics is, however, frustrated by the small number of high-energy neutron irradiation SEE test facilities. The need to test is further exasperated as the continued miniaturization of state-of-the-art electronics has brought an increased sensitivity of digital devices to Soft Errors such as SEU induced by neutrons with energies below $10 \mathrm{MeV}$.

\section{ANEM: The Atmospheric-Neutron EMulator}

A new variable-energy, high-current proton cyclotron $(35-70 \mathrm{MeV} ; 0.75 \mathrm{~mA})$ is being commissioned at the LNL SPES facility ${ }^{5,6}$ in Legnaro, Italy. The SPES proton beam will also be used to feed a relatively inexpensive and accessible neutron and proton irradiation facility (NEPIR). ${ }^{7,8}$ One of the NEPIR beam lines will use the Atmospheric Neutron Emulator (ANEM), a specialized neutron production target for studying SEE induced by atmospheric neutrons. It will produce a continuous energy neutron beam with an energy distribution similar to that of atmospheric neutrons found at flight-altitudes and at sea level in the 1-70 MeV energy range. The NEPIR facility foresees also a multi-purpose Quasi Mono-Energetic (QMN) beam with a controllable discrete energy peak in the 20 $70 \mathrm{MeV}$ range. ${ }^{9}$

The ANEM system is a rotating composite target made of beryllium, $24 \mathrm{~mm}$ thick, and tungsten, $5 \mathrm{~mm}$ thick, shaped into two separate circular sectors that alternatively intercept the proton beam (Fig. 2). The sectors with monolithic coaxial rings are mounted on a rotating steel drum with a $20 \mathrm{~cm}$ diameter cooled by a helicoidal water cooling system (Fig. 3). The thermal contact of the sectors with the drum is ensured by a $2 \mathrm{~cm}$ thick copper ring sandwiched between the two sectors by steel screws.
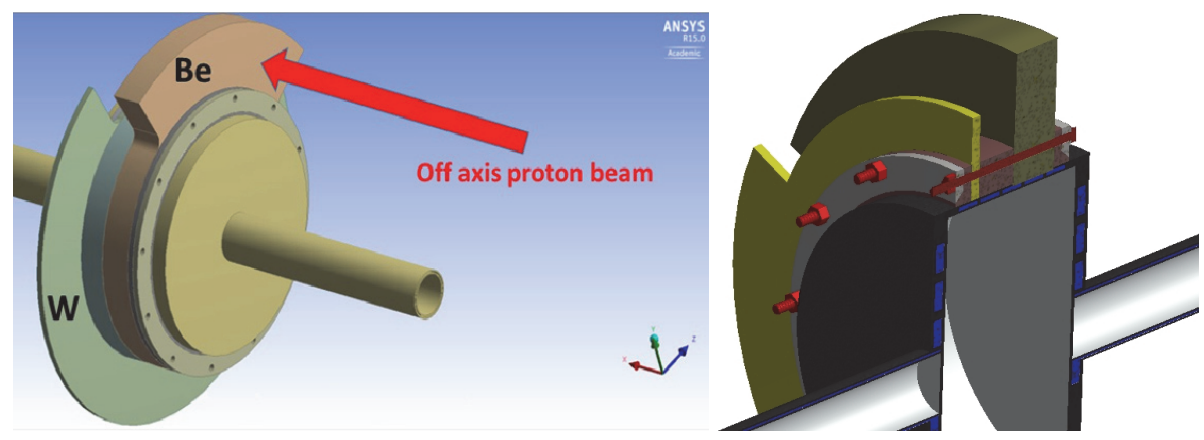

Fig. 2. The $32 \mathrm{~cm}$ diameter Be and $\mathrm{W}$ circular sectors of ANEM alternatively intercept the $13 \mathrm{~cm}$ off-axis proton beam (left); the stainless steel rotating drum (diameter $18 \mathrm{~cm}$ ) houses the helicoidal water cooling circuit (right). In brown the $\mathrm{Cu}$ flange for heat removal.

The thickness of the $\mathrm{W}$ target has been chosen in order to fully contain the proton beam. On the contrary, the proton beam must exit the Be target to avoid blistering caused 
by hydrogen buildup. The spent $\sim 20 \mathrm{MeV}$ protons emerging from the Be are then stopped by a $1 \mathrm{~mm}$ thick copper plate located downstream.

The effective neutron spectrum is shaped to resemble the atmospheric one (up to the cyclotron energy) by optimizing the relative areas of the two sectors, without the use of moderators. The optimal percentage of $\mathrm{Be}$ to $\mathrm{W}$ to ensure the best atmospheric-like shape is determined via Monte Carlo (MC) code simulations: the W reproduces the lower energy part (few $\mathrm{MeV}$ ) of the atmospheric neutron spectrum while Be provides more high energy neutrons. Fig. 4 shows the simulated effective fast neutron differential energy spectrum (MCNPX) ${ }^{10}$ produced by ANEM at a downstream distance of $6 \mathrm{~m}$ for a proton current $I_{p}=30 \mu \mathrm{A}$ and energy $E_{p}=70 \mathrm{MeV}$. In this case, the Be sector takes up $18 \%$ of the full circle.

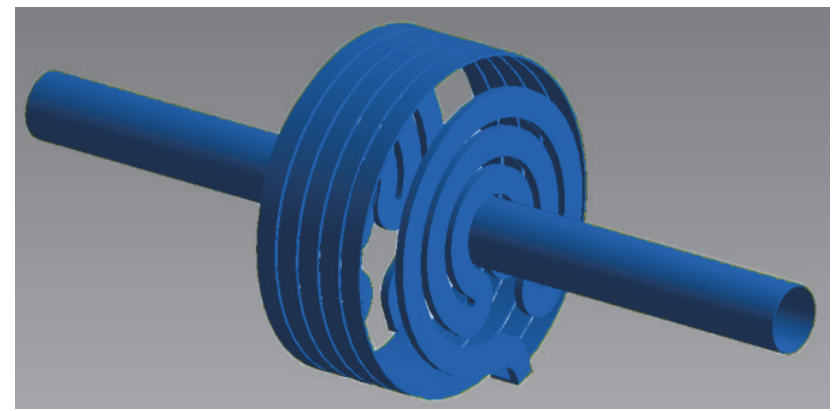

Fig. 3. The the helicoidal water cooling circuit.

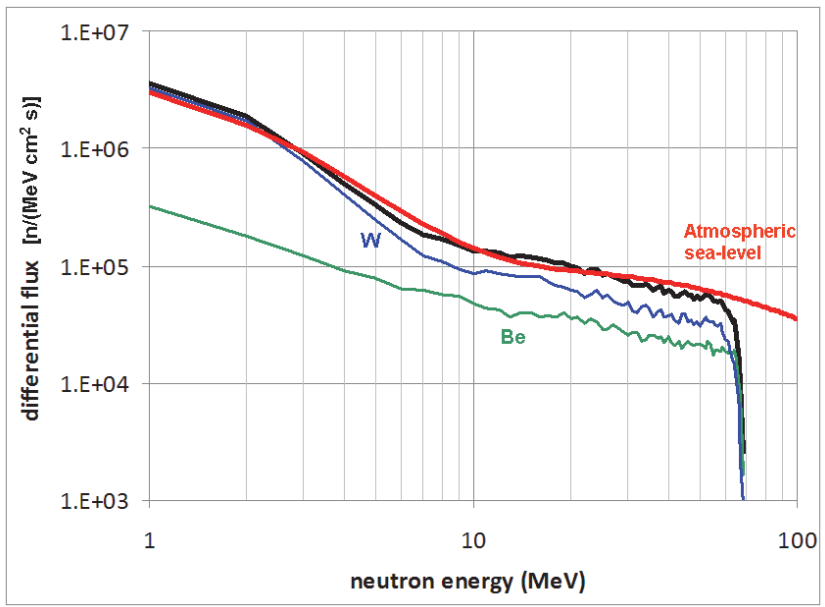

Fig. 4. The ANEM neutron spectrum (black) with $70 \mathrm{MeV}$ protons and $30 \mu \mathrm{A}$ current at a distance $6 \mathrm{~m}$ downstream, compared to the reference sea-level one (red) multiplied by an acceleration factor $\mathrm{F}=3 \times 10^{9}$. The ANEM spectrum is the weighted sum of the spectra of the two sectors: Be $18 \%$ (green curve), W $82 \%$ (blue curve).

In this energy range, the proton-on-W neutron spectra are well modeled with updated libraries; however, Be is not, and we are comparing MC codes (MCNPX, FLUKA ${ }^{11}$, 
PHITS $^{12}$ ). At the moment of submission of this contribution, the optimal amount of Be is still too uncertain (the amount of $\mathrm{Be}$ is between $\sim 20-40 \%$ ). Before the construction of the final prototype, we plan to measure with a dedicated experiment the neutron spectrum of $70 \mathrm{MeV}$ protons on $24 \mathrm{~mm}$ thick Be (backed with a proton stopping $\mathrm{Cu}$ plate). In the following, we assume that the Be sector takes up $18 \%$ of the full circle.

\section{Finite Element Analysis with ANSYS}

Figure of merit of ANEM is the high acceleration factor $\mathrm{F}=3 \times 10^{9}$. Such a value can be obtained using approximately $\sim 5 \%$ of the available proton current $(700 \mu \mathrm{A})$. A current of $30 \mu \mathrm{A}$ of $70 \mathrm{MeV}$ protons delivers a power of $2.1 \mathrm{~kW}$ in a small volume. It is hence mandatory to calculate the temperature map of the target materials and to evaluate the mechanical stress.

We used ANSYS Workbench Platform ${ }^{13}$ to perform an extensive series of calculations to optimize the target design, modeling its thermo-mechanical behavior. The ANSYS CFX module was used to model the thermal power propagation from the irradiated area of the sectors down to the water-cooling system.

To estimate the regime temperature reached by the different sectors of the target during operation, we modeled the heat deposited by the beam impinging on the rotating target as a volume heat source. The thermal power deposition in the irradiated volumes of the Be and $\mathrm{W}$ sectors were described with increasing levels of accuracy until the model reached a sufficient refinement not to gain precision by further improvement.

We started by assuming the energy delivered by the proton beam as spread over an annular sector, as if the target was rotating with an infinite speed. The beam distribution inside this area was first assumed to be uniform and then to have a Gaussian shape $(\mathrm{FWHM}=1 \mathrm{~cm})$ with the maximum in the middle of the annulus (Fig. 5a). This approach neglects the local temperature variations induced by a pencil-like beam striking a point on the rotating target and estimates only the time-averaged temperature. We just recently started to study the effects of this time-dependent periodic behavior of the temperature distribution.

Initially the energy deposition along the proton beam direction was assumed to be uniform and then to have a non-constant value according to the Bethe-Bloch distribution (Fig. 5). The distribution was calculated using $\mathrm{SRIM}^{14}$ and was approximated by dividing each of the target sectors into 3 slices, along the beam direction, and by calculating the amounts of energy deposited in each slice.

Unlike the $\mathrm{W}$ sector that is beam-stopping, the Be sector is thin enough to allow the proton beam to exit before reaching the maximum of the Bragg peak, and only $71 \%$ of the beam energy is absorbed. The description of the energy deposition did not include the lateral spreading of the beam in the target, resulting in a more concentrated energy deposition (effectively a worst case scenario).

Figure 6 shows the temperature distribution for a total delivered power $\left(P=I_{p} \times E_{p}\right)$ of $2.5 \mathrm{~kW}$. The beam shape is Gaussian and the energy deposition is according to approximation of the Bethe-Bloch distribution described above. The maximum 
temperature as a function of the total delivered power is shown in Fig. 7. The maximum scales linearly with power, down to very low power values.
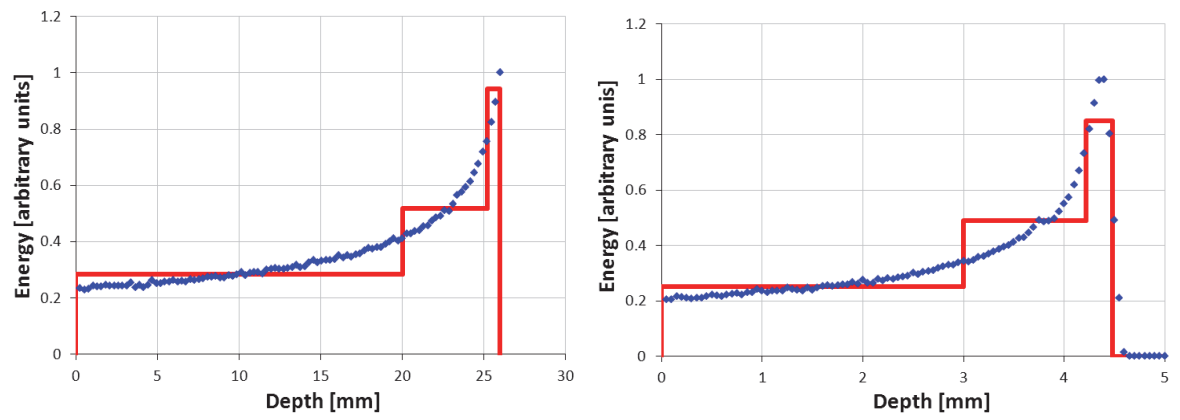

Fig. 5. The Linear Energy Transfer distribution in Be (left) and W (right) calculated using SRIM (blue dots). In red the step-wise approximation used in the ANSYS calculations.

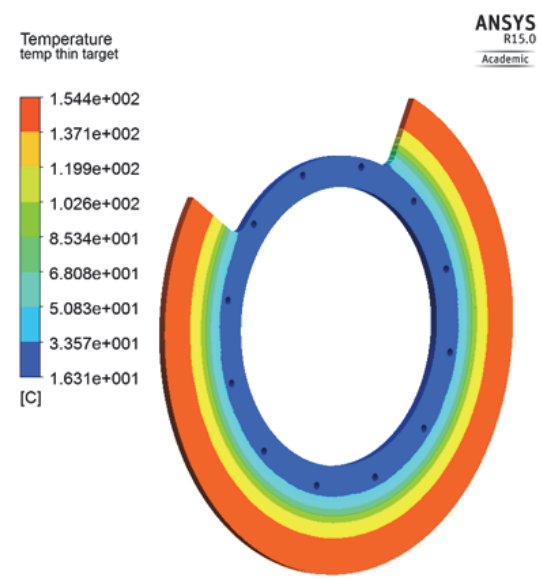

Fig. 6. Temperature map of the $\mathrm{W}$ sector model for a power deposition of $2.5 \mathrm{~kW}$. Other conditions are dectibed in the text.

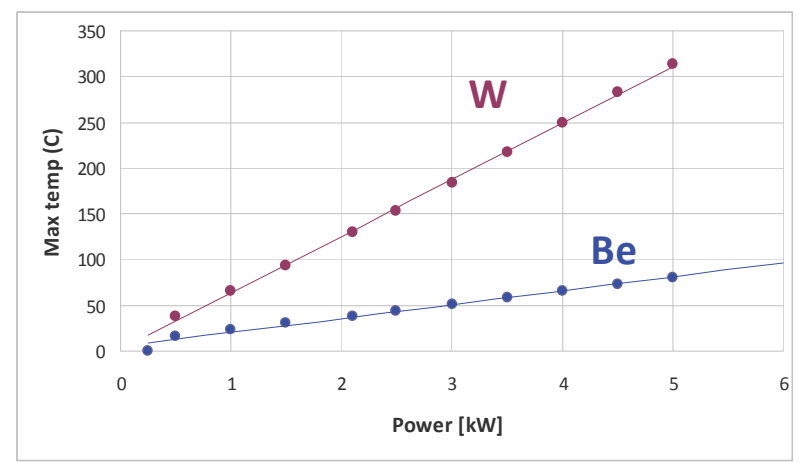

Fig. 7. The maximum temperature reached by the $\mathrm{Be}$ and the $\mathrm{W}$ sectors as a function of the total delivered power. 
The calculated thermal maps were then used by the ANSYS Structural module to obtain the distribution of the stress for each element (Be and W). Figure 8 shows the stress distribution for the thermal conditions described in Fig. 6.

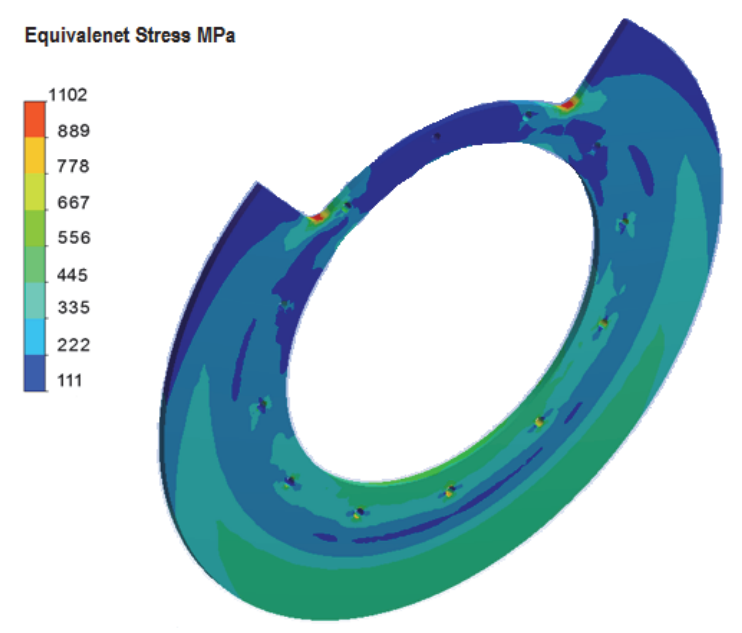

Fig. 8. Stress map of the $\mathrm{W}$ sector model for a power deposition of $2.5 \mathrm{~kW}$.

To evaluate the robustness of the target, the maximum stress was compared with the temperature dependent yield strength of the material as a function of the delivered power (Fig. 9). For an extra safety margin, the comparison is with the $50 \%$ value of the yield strength. The maximum stress of the $\mathrm{W}$ sector scales linearly with power, and it crosses the $50 \%$ yield strength curve at $2.3 \mathrm{~kW}$. The maximum stress of Be sector also scales linearly but for power values higher than $2.5 \mathrm{~kW}$ and is almost constant at low power vales, presumably because of the stress induced by the steel screws dominates. More importantly, the maximum stress in Be exceeds the $50 \%$ yield strength value only for very high power values ( $>6 \mathrm{~kW})$ (Fig. 9).
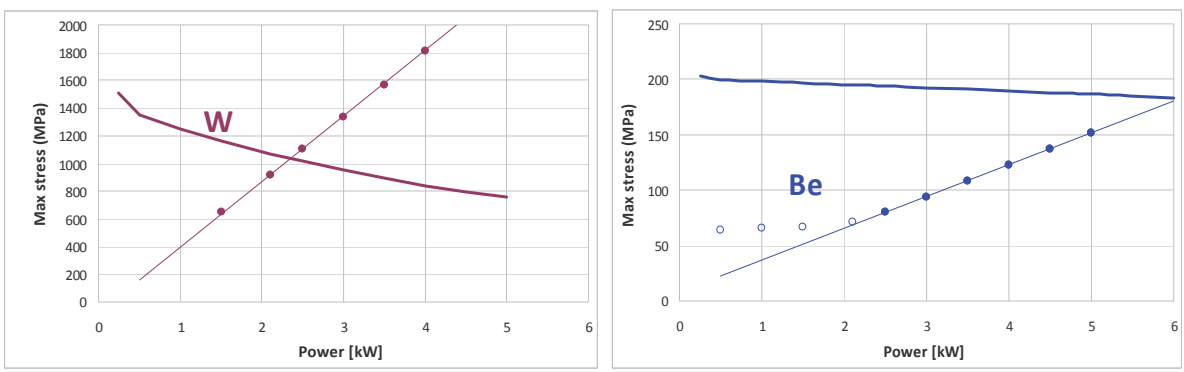

Fig. 9. The maximum stress (circles) in each target was calculated using ANSYS for different values of delivered power. The thick lines in both figures are the temperature dependent yield strengths multiplied by a safety factor of $50 \%$. 
The power dissipation capabilities of the ANEM prototype will be experimentally tested over the next semester, mimicking the proton beam with a $10 \mathrm{keV}$ electron gun, 1 A maximum current. Aluminum dummy sectors will be used instead of the final Be and W sectors. The test will be used to validate and tune an improved ANSYS model that will include the time-dependent behavior due to a rotating pencil beam. Such a model will lead the final design of ANEM.

\section{Conclusion}

MC simulations show that ANEM will be able to deliver, at a distance of $6 \mathrm{~m}$ from the target, an atmospheric-like beam of neutrons in the 1-70 MeV energy range with a maximum integral flux up to $\Phi_{\mathrm{n}}(1-70 \mathrm{MeV}) \sim 10^{7} \mathrm{n} \mathrm{cm}^{-2} \mathrm{~s}^{-1}-\mathrm{F}=3 \times 10^{9}$ more intense than the natural one at sea level in the same energy range. This can be achieved with the SPES cyclotron operating at full energy $(70 \mathrm{MeV})$ with a proton current of $30 \mu \mathrm{A}$. The ANSYS modelling described in this paper indicates that the ANEM prototype can conservatively handle the $2.1 \mathrm{~kW}$ delivered by such a beam.

Such high neutron fluxes make it possible to study Single Event Effects with small cross-sections. The ability to control the proton beam current will allow the user to irradiate devices with large SEE cross-sections avoiding pile-up effects.

The ANEM facility at LNL should prove to be strategic for high-tech industries that develop devices and systems for various high-reliability applications (industrial automation, information technology, automotive, biomedical, security, military, etc.). These industries need to check their innovative products for unexpected sensitivity to lower energy atmospheric neutrons, especially in the development and engineering phases before final certification.

\section{References}

1. T. Nakamura, M. Baba, E. Ibe and Y. Yahigi, Terrestrial Neutron-Induced Soft Errors in Advanced Memory Devices (World Scientific Publishing Co. Pte. Ltd, 2008).

2. JESD89A, Measurement and Reporting of Alpha Particle and Terrestrial Cosmic Ray-Induced Soft Errors in Semiconductor Devices, Oct. 2006.

3. A. V. Prokofiev et al., Characterization of the ANITA Neutron Source for Accelerated SEE Testing at The Svedberg Laboratory, in 2009 IEEE Radiation Effects Data Workshop (Quebec, Canada, July 20-24, 2009), pp. 166-173.

4. http://www.isis.stfc.ac.uk/instruments/chipir/

5. SPES Technical Design Report, 2008, eds. G. Prete and A. Covello INFN-LNL223 http://www.lnl.infn.it/spes/TDR2008/tech_design08_index.htm; http://www.bestcyclotron.com/product_70p.html.

6. Gianfranco Prete, A. Andrighetto, J. Esposito, P. Mastinu and J. Wyss, Physics Procedia 26, 274 (2012).

7. D. Bisello et al., Physics Procedia, 60, 66 (2014).

8. D. Bisello et al., Physics Procedia 26, 284 (2012).

9. D. Bisello et al., Physics Procedia 60, 271 (2014).

10. D. B. Pelowitz, M. R. James, G. W. McKinney, J. W. Durkee et al., MCNPX 2.7.A Extensions (LANL, 2008). 
11. A. Ferrari, P.R. Sala, A. Fasso` and J. Ranft, FLUKA: a multi-particle transport code, CERN2005-10 (2005), INFN/TC_05/11, SLAC-R-773.

12. T. Sato et al., Particle and Heavy Ion Transport Code System PHITS, Version 2.52, J. Nucl. Sci. Technol. 50:9, 913 (2013).

13. ANSYS ${ }^{\circledR}$ Academic Research, Release 15.0.

14. SRIM 2013; www.srim.org. 